\title{
Emphysematous Pyelonephritis in Two Cases at Two Different Stages: Early and Late
}

\author{
Aslan Demira, c, Kursat Cecen ${ }^{\mathrm{a}}$, Mert Ali Karadaga, Eray Atalay, \\ Mehmet Uslua, Omer Erkam Arslan ${ }^{\mathrm{a}}$
}

\begin{abstract}
Emphysematous pyelonephritis (EPN) has been defined as a necrotizing infection of the renal parenchyma and its surrounding areas that result in the presence of gas in the renal parenchyma, collecting system or perinephric tissue. The final diagnosis mostly depends on the radiological findings since clinical and biochemical findings of the EPN can be different from case to case. The diseases can be seen not only in diabetic patients but also occasionally in patients without diabetes mellitus with obstruction of the the urinary system. In this article, we discussed the EPN based on two patients at the two different stages, late and early, respectively.
\end{abstract}

Keywords: Emphysematous pyelonephritis; Nephrectomy; Clinical stages; Medical treatment

\section{Introduction}

Emphysematous pyelonephritis (EPN) has been defined as a necrotizing infection of the renal parenchyma and its surrounding areas that result in the presence of gas in the renal parenchyma, collecting system or perinephric tissue [1]. This disease was first described by Kelly and MacCullem in 1898 [2], while the term EPN was first used by Schultz and Klorfein in 1962 [3]. Unfortunately, except a few studies based on the evidence-based approach, most of the information about EPN in the literature has been presented as case reports [4]. EPN is much more seen in females, with various

\footnotetext{
Manuscript accepted for publication May 6, 2014

${ }^{a}$ Urology Department, School of Medicine, Kafkas University, Kars, Turkey

b Internal Diseases Department, School of Medicine, Kafkas University, Kars, Turkey

${ }^{\mathrm{c}}$ Corresponding Author: Aslan Demir, Kafkas University, Tip Fakultesi

Hastanesi, Uroloji A.B.D, Kars, Turkey.

Email: draslandemir@yahoo.com
}

doi: http://dx.doi.org/10.14740/jmc1799w studies reporting the female to male ranging from $3: 1$ to $43: 3$ $[5,6]$.

The final diagnosis mostly depends on the radiological findings since clinical and biochemical findings of the EPN can be different from case to case. The diseases can be seen not only in diabetic patients but also occasionally in patients without diabetes mellitus (DM) with obstruction of the urinary system. The severity of the diseases may depend on the co-morbidities of the patients such as DM, urinary system obstruction with or without stones and perhaps the time of the applying to the doctor and true diagnosis time after the beginning of the disease.

In this article, we discussed the EPN based on two patients at the two different stages, late and early, respectively.

\section{Case Report}

\section{Case 1}

A 69-year-old male patient was admitted to our clinic with left flank pain, high fever $\left(39^{\circ} \mathrm{C}\right)$, vomitting, dysuria and vague abdominal pain. He had a co-morbiditiy such as DM that is not under control for 3 years. He had a left nephrolitotomy history 10 years ago. The abdominal examination was normal and costo-vertebral tenderness in his left side was positive in his physical examination. General health status was at the low level due to insufficient oral intake at the beginning of hospitalization. We established leukocyto-

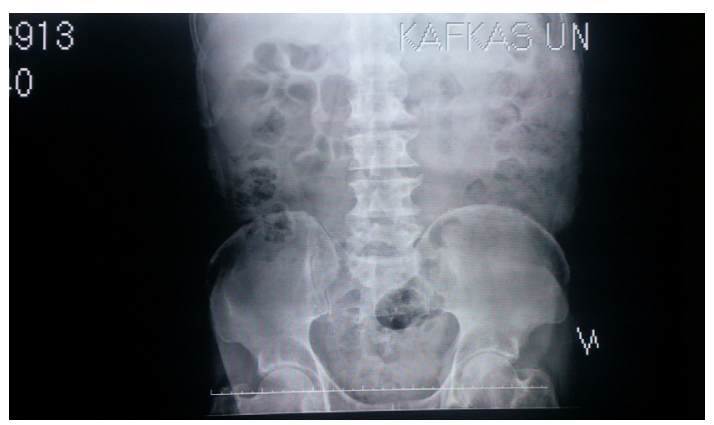

Figure 1. The plain abdominal graphy for case 1. 

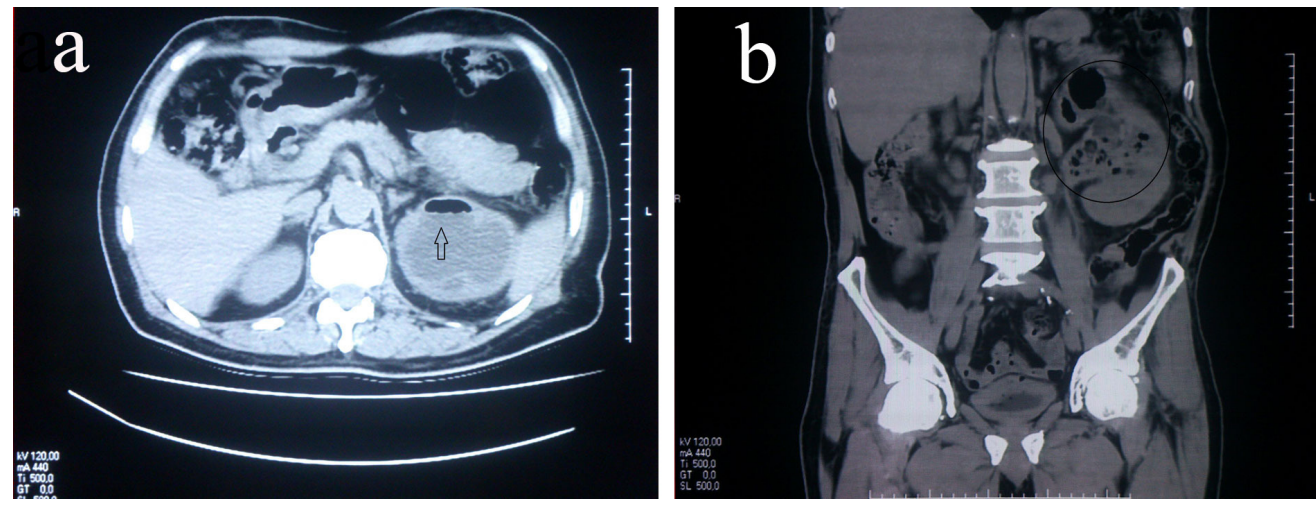

Figure 2. The computed tomography belonging to case 1, transverse (a) and coronal (b) sections, respectively.

sis as 11,410 counts, hemoglobine $13.0 \mathrm{~g} / \mathrm{dL}$ and plateletes $3,910,000 / \mathrm{mm}^{3}$ in his blood analysis, 509 white blood cells (WBC) and 6 red blood cells (RBC), glucosuria were established in his urine analysis. It was not established any electrolyte imbalance in his blood analysis, creatinine was 1.09 , BUN was 27, glucose in his blood was 129 , CRP was 29, and sedimentation was 50 . Urine culture was sterile. There was intense gas image in his plain abdominal graphy (Fig. 1). The whole abdominal computed tomography was applied before ultrasonography because of the intense gas image in his plain abdominal X-ray and established hypertrophy and the intense gas in the renal paranchyma and its outside (Fig. $2 \mathrm{a}, \mathrm{b})$. The final diagnosis was concluded as EPN with these findings. The broad-spectrum antibiotic was applied in his medical treatment as well as intravenous fluid support with conservative treatment. Although the antibiotic therapy was given, the left nephrectomy was applied because of the deterioration of the clinic course of the patient and his labratory findings at the fifth day of the treatment. The extent purulant material was observed macroscopically in the nephrectomy specimen and it was reported Candida albicans microscopically and the extent cortical necrosis, acut chronic pyelonephritis in the pathologic specimen. The amelioration of the clinic was observed in the post-operative follow-up.

\section{Case 2}

A 37-year-old female patient was hospitalized with a right flank pain, dysuria, high fever, abdominal pain and general health disorders because of the decreasing of the oral intake. It could not be established any co-morbidities in her health history except constipation. Her physical examination revealed a right costo-vertebral tenderness, high fever (38.5 ${ }^{\circ} \mathrm{C}$ ), hypotension and abdominal tenderness which had not acute abdominal findings. Pyuria was found in her urine analysis and her blood analysis revealed electrolyte imbalance (Na: $132 \mathrm{mmol} / \mathrm{L}, \mathrm{K}^{+}: 2.77 \mathrm{mmol} / \mathrm{L}$ ), high level hepatic enzymes (AST: $78 \mathrm{U} / \mathrm{L}$, ALT: $74 \mathrm{U} / \mathrm{L}$ ), normal level blood
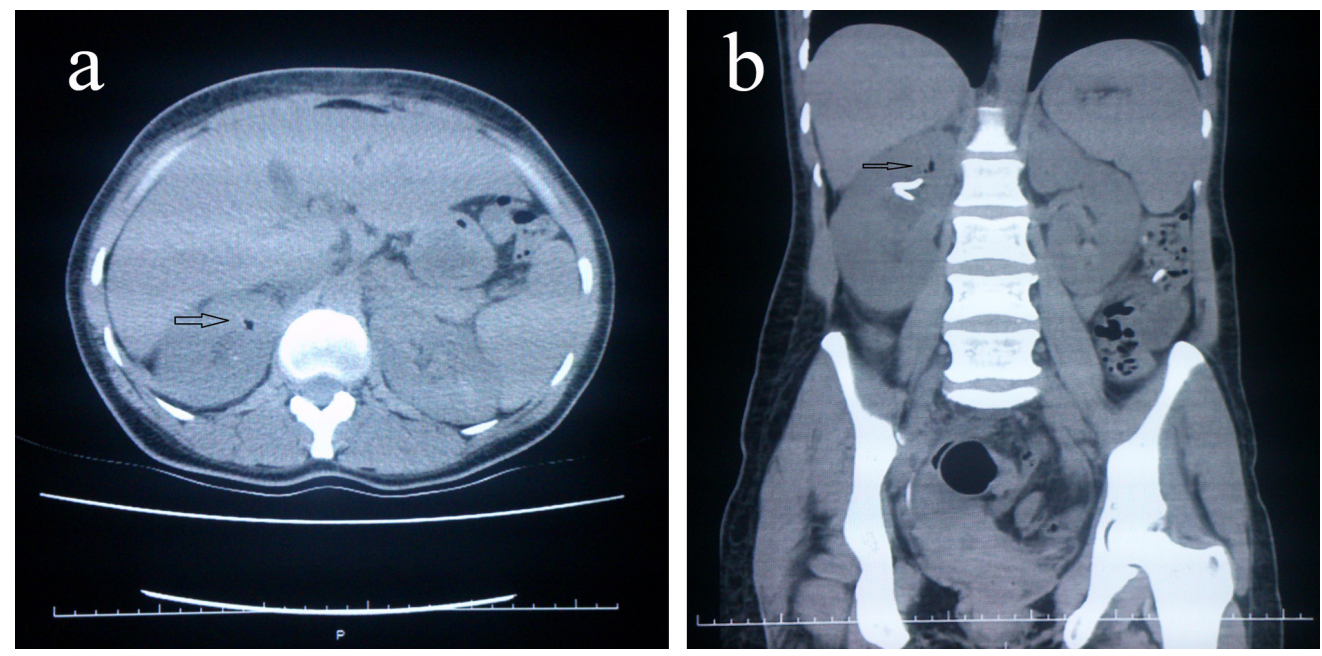

Figure 3. The computed tomography belonging to case 2. (a) Pre-treatment, the formed gas in the renal parenchyma (in transverse section). (b) In coronal section, including $6 \mathrm{Fr}$ double $\mathrm{J}$ stent in the renal pelvis. 
urea nitrogen and a bit low level creatinine $(0.6 \mathrm{mg} / \mathrm{dL})$, high level glucose at the starvation $(224 \mathrm{mg} / \mathrm{dL})$, WBC: 12,000 , platelets: 350,000 , high level CRP $(31.6 \mathrm{mg} / \mathrm{dL})$ and sedimentation (85). Blood culture (aerob and anerob) that was taken during high fever and urine culture were investigated microbiologycally. The $6 \mathrm{Fr}$ double J stent was inserted into her right kidney because it was reported that she had dilatation on her right kidney according to the urinary system ultrasonography and broad spectrum antibiotics were given as well as intravenous hydration and conservative treatment. The computed tomography was performed due to late response to the applied treatment at the third day of her hospitalization and this revealed paranchymal localized gas in the right kidney (Fig. 3a, b). The final diagnosis was EPN after CT findings. Three kinds of bacterias that were cultured in her urine analysis were depended on contamination. No bacteria could be cultured in her blood culture. Her whole clinical, labratory and radiological findings were normal at tenth day of the treatment. The applied treatment was terminated at the 14th day of the treatment. It could not be established any disorders during follow-up for 3 months.

\section{Discussion}

Gas-forming infection of the kidneys can affect either the parenchyma or the collecting system, the perinephric tissue and carries a bad prognosis $[4,7,8]$. They are named as EPN or emphysematous pyelitis (EP) according to the place where the infection is $[4,7]$. The clinical course of EPN can be severe and life-threatening if not recognized and treated promptly. A patient infected with EP has an excellent prognosis with medical management, whereas EPN deserves special attention because of its life-threatening potential with either medical management (MM) or surgical treatment. Mortality from EPN is primarily attibutable to septic complications. EPN was associated with a mortality rate of up to $78 \%$ until the late 1970s but, over the last two decades, improvement in management techniques has reduced the mortality rate to $21 \%[9,10]$. Most of the information has been from case reports, and a few large series have also been reported [4].

There is a preponderance of EPN in females; the female male ratio reported in relatively small studies is $6: 1,3: 1$ or $43: 3[1,5,6,10,11]$. Increased susceptibility to urinary tract infection seems to be the reason for the high incidence in females; also in our opinion, constipation which is mostly related with sociaeconomic status and nutritional habitus may increase the risk in females like in the second case that we presented. The risk of developing EPN secondary to a urinary tract obstruction is about $25-45 \%[12,13]$.

$\mathrm{DM}$ is the single most common associated factor. Up to 95\% of patients with EPN have underlying uncontrolled DM $[9,11]$. The presence of DM appeared to be a common risk factor for EPN but, suprisingly, it is not associated with in- creased mortality [11]. The first case that we presented had also uncontrolled DM and the other one had glucose irregularity which ameliorated with MM. Other reported factors associated with the development EPN are drug abuse, neurogenic bladder, alcoholism and anatomic anomalies [12-15].

Huang and Tseng have postulated that four factors are involved in the pathogenesis of EPN, which were gasforming bacteria, high tissue glucose level, impaired tissue perfusion and a defective immun response [1]. Leukocyte dysfunction seen in diabetics may contribute to the pathogenesis of EPN. Gas-production in the renal parenchyma in the absence of infection has also been described following traumatic renal infarction [16]. The infection organisms are usually glucose-fermenting bacteria. E. coli is the most common bacteria implicated in EPN, others are Klebsiella and proteus mirabilis. EPN caused by group D streptococcus, coagulase-negative staphylococcus and anerobic microorganisms including Clostridium septicum, Candida albicans, Criptococcus neoformans and Pneumocystis Jiraveci have, in rare cases, been reported $[9,17,18]$. The presence of a less common causative organism in urine or blood culture does not require that patient to be treated for EPN unless there are radiological features to suggest that EPN might be present [4]. In just the same way, no any bacteria was cultured in either urine nor blood culture in the first case that we reported. That's why, we performed left nephrectomy both depending on the finding of CT and due to no response to the treatment that we applied at the fifth day and Candida albicans was cultured at purulant material that was taken from the nephrectomy specimen in the first case that we reported. Although three kinds of bacteria that were cultured in urine of the second case, the accurate-final diagnosis was accepted as EPN by depending on the gas in her left kidney parenchyma in her CT finding (Fig. 3a, b).

The clinical presentation is often suggestive of severe acute pyelonephritis, with fever, flank pain and pyuria being the most common clinical manifestations. However, these are non-spesific and may be seen in other forms of upper urinary tract infections. Other clinical features of EPN include non-spesific abdominal pain, nausea, vomiting, depressed levels of consciousness, shock, renal angle tenderness, dysuria, pneumaturia and crepitations in the flanks but rarely extenting to the scrotum $[1,3,5,6,18,19]$. The clinical presentations of the cases that we reported are similar with the literature except crepitation sign. Their general health status was bad due to the insufficient oral intake. We evaluated the patients with the urine analysis, plain abdominal X-ray and urinary sistem ultrasonograpy as well as blood tests at the hospitalization. We could not determine any special findings related to EPN. The second case was more attractive because of the abnormality in her blood tests such as electrolyte imbalance and glucose irregularity altough she had not any DM in her health history. Her HbAlc level was established as normal. The reason of her electrolyte imbalence may be as- 
sociated with decreasing of her oral intake or intense infection, in addition to this it can be a message for pre-shock status. There was no trombocytopenia in our cases in contrast with some cases that have been presented in the literature. In the Huang and Tseng study, trombocytopenia (46\%), acute renal function impairment (35\%), disturbance of consciousness $(19 \%)$ and shock $(29 \%)$ can be the initial presentations [1]. Shokier et al found renal function impairment in $80 \%$ of their patients and shock and coma in $15 \%$ of patients [6]. In addition to these, no significant association could be established between higher mortality in EPN and nephrolithiaisis, E. coli or Klepsiella pneumonia etiology of EPN, age $>50$ years old, female sex, history of UTI or alcoholism [4]. Systolic blood pressure less than $90 \mathrm{~mm} \mathrm{Hg}$, disturbance of consciousness as well as increase in serum creatinine level were found to be associated with higher mortality. The presence of thrombocytopenia and bilateral EPN are both associated with poor prognosis. Medical management with antibiotics alone is associated with higher risk of mortality when compared with additional interventions of percutaneous drainage of the abscess or nephrectomy [11].

EPN is a radiological diagnosis because of the other nonspecific findings. To evaluate such a patient, plain abdominal $\mathrm{X}$-ray and ultrasonography is mandatory and helpful but the results can not be enough for EPN diagnosis. Ultrasonography and plain radiograph of the abdomen are only accurate in 69 and $65 \%$ of cases, respectively [10]. If there is intense gas in a patient, the effectivity of ultrasonograhy and plain abdominal X-ray will be low for detection of EPN. CT will confirm the presence of intrarenal gas which supports the diagnosis of EPN. So, abdominal CT is neccessary for early diagnosis and further management of EPN [10], especially, in the cases that response lately to the applied treatment.

The CT is really important not only for accurate diagnosis but also for the desicion for further management at the follow-up. Contrast-enhanced CT scan is better as it gives an idea about the function status of the renal unit as well as it facilitates the description of the intraparenchymal gas (streaky, mottled, bubbly, rimlike, cresent shaped, locular, etc.). However, in patients with deranged renal parameters, a non-contrast $\mathrm{CT}$ scan may suffice. In addition to diagnosis and staging of EPN, CT scan is also helpful in monitoring the response to treatment. It might show resolution of the gas and abcesses or the development of new lesions [20]. Wan et al classified the gas collection as type I or type II, on the basis of CT scans. Type I: Renal necrosis with presence of gas but no fluid. Type II: Parenchymal gas associated with fluid in renal parenchyma, perinephric space or collecting system. Mortality rates in type I and II were $69 \%$ and $18 \%$, respectively [9]. Similar mortality rates were observed by Chen et al [21]. Huang and Tseng also used CT to classify patients with EPN as follows [1]: Class I: Gas in collecting system only. Class II: Parenchymal gas only. Class III-A: Extension of gas into perinephric space. Class III-B: Exten- sion of gas into pararenal space. Class IV: Bilateral disease or EPN in solitary kidney.

The classification by Huang and Tseng is superior due to the better prognostic value and also helpful in selecting a management protocol. In their study, class I and II patients, all survived following treatment with percutaneous procedures and medical therapy. While in patients belonging to class III or IV, those with fewer than two risk factors (i.e. trombocytopenia, acute renal function impairment, disturbance of consciousness and shock) had an $85 \%$ survival rate with percutaneous drainage (PCD) and medical therapy, whereas patients of class III or IV EPN and two or more risk factors had a $92 \%$ failure rate with PCD and medical therapy [1]. The proportion of non-responders in this group requiring nephrectomy is higher compared with class I and II EPN [1, 18]. Kapoor et al also investigated the predictive factors for mortality and need for nephrectomy in patients with EPN and concluded that altered mental status, thrombocytopenia, renal failure and severe hyponatremia at presentation were significantly associated with mortality rate. There was no significant difference in final outcome based on radiological classification. Extensive renal paranchymal destruction of $>$ $50 \%$ (based on CT) significantly predicted the need for nephrectomy and death. Early ( $<1$ week) nephrectomies carry higher mortality rates than initial conservative management [22]. We also performed nephrectomy at the fifth day of the treatment in our first case, because of the deterioration of patient's clinic and CT findings that revealed extensive renal parenchymal destruction $>50 \%$ (Fig. 2a, b).

In patients, who are being treated for pyelonephritis, the radiological diagnosis may be missed, unless appropriate imaging is obtained. This group of patients, along with those who fail to respond to standard line of treatment of pyelonephritis, should have an urgent CT scan to confirm the diagnosis [4]. Conservative treatment according to the patient situation should be started immediately and the antibiotics that will be applied should include gram negative bacteria unless a special bacteria is cultured. If so, then the antibiotics can be changed according to culture-antibiogram report. The patients should be evaluated again and again during hospitalization according to the clinical, labratory and radiological findings. If neccesary, it should not be avoided to insert any method for drainage of upper urinary system (percutaneous catheter or double J stent) or nephrectomy. In a meta-analysis of the management strategies, the most successful management was MM with PCD (30-100\%), which was also associated with the lowest mortality at $13.5 \%$ [10]. In a small proportion of patients managed with $\mathrm{MM}$ and PCD, subsequent nephrectomy will be required and in these patients the reported mortality is $6.6 \%[10]$.

According to the current literature, different clinical status may be related to stage of the disease or sometimes host factors such as co-morbidities. The EPN has been mostly observed in crowded populations when we search which coun- 
try has mostly published about it. That's why, we believe that according to this search, environmental, nutritional, socioeconomic and sociocultural factors should be taken into account for EPN.

\section{Conflict of Interest}

None.

\section{References}

1. Huang JJ, Tseng CC. Emphysematous pyelonephritis: clinicoradiological classification, management, prognosis, and pathogenesis. Arch Intern Med. 2000;160(6):797-805.

2. Kelly HA, MacCullem WG. Pneumaturia. JAMA. 1898;31:375.

3. Schultz EH, Jr., Klorfein EH. Emphysematous pyelonephritis. J Urol. 1962;87:762-766.

4. Ubee SS, McGlynn L, Fordham M. Emphysematous pyelonephritis. BJU Int. 2011;107(9):1474-1478.

5. Pontin AR, Barnes RD. Current management of emphysematous pyelonephritis. Nat Rev Urol. 2009;6(5):272279.

6. Shokeir AA, El-Azab M, Mohsen T, El-Diasty T. Emphysematous pyelonephritis: a 15-year experience with 20 cases. Urology. 1997;49(3):343-346.

7. Tan CCKHGH, Bahadzor B, Praveen S, Goh EH, Syahril AS, Zulkifli MZ. Bilateral emphysematous pyelitis: a rare encounter in urology. Clin Ter. 2013;164(4):319-321.

8. Mohsin N, Budruddin M, Lala S, Al-Taie S. Emphysematous pyelonephritis: a case report series of four patients with review of literature. Ren Fail. 2009;31(7):597-601.

9. Wan YL, Lee TY, Bullard MJ, Tsai CC. Acute gas-producing bacterial renal infection: correlation between imaging findings and clinical outcome. Radiology. 1996;198(2):433-438.

10. Somani BK, Nabi G, Thorpe P, Hussey J, Cook J, N'Dow J. Is percutaneous drainage the new gold standard in the management of emphysematous pyelonephritis? Evidence from a systematic review. J Urol.
2008;179(5):1844-1849

11. Falagas ME, Alexiou VG, Giannopoulou KP, Siempos, II. Risk factors for mortality in patients with emphysematous pyelonephritis: a meta-analysis. J Urol. 2007;178(3 Pt 1):880-885; quiz 1129.

12. Michaeli J, Mogle P, Perlberg S, Heiman S, Caine M. Emphysematous pyelonephritis. J Urol. 1984;131(2):203208.

13. Godec CJ, Cass AS, Berkseth R. Emphysematous pyelonephritis in a solitary kidney. J Urol. 1980;124(1):119121.

14. Morehouse HT, Weiner SN, Hoffman JC. Imaging in inflammatory disease of the kidney. AJR Am J Roentgenol. 1984;143(1):135-141.

15. Abdul-Halim H, Kehinde EO, Abdeen S, Lashin I, AlHunayan AA, Al-Awadi KA. Severe emphysematous pyelonephritis in diabetic patients: diagnosis and aspects of surgical management. Urol Int. 2005;75(2):123-128.

16. Subramanyam BR, Lefleur RS, Van Natta FC. Renal emphysema secondary to traumatic renal infarction. Urol Radiol. 1980;2(1):53-54.

17. Hildebrand TS, Nibbe L, Frei U, Schindler R. Bilateral emphysematous pyelonephritis caused by Candida infection. Am J Kidney Dis. 1999;33(2):E10.

18. Khaira A, Gupta A, Rana DS, Bhalla A, Khullar D. Retrospective analysis of clinical profile prognostic factors and outcomes of 19 patients of emphysematous pyelonephritis. Int Urol Nephrol. 2009;41(4):959-966.

19. Kumar VS, Lakshmi AY. Emphysematous pyelonephritis. Indian J Nephro. 2004;14:192-194.

20. Sharma PK, Sharma R, Vijay MK, Tiwari P, Goel A, Kundu AK. Emphysematous pyelonephritis: Our experience with conservative management in 14 cases. Urol Ann. 2013;5(3):157-162.

21. Chen MT, Huang CN, Chou YH, Huang CH, Chiang CP, Liu GC. Percutaneous drainage in the treatment of emphysematous pyelonephritis: 10-year experience. J Urol. 1997;157(5):1569-1573.

22. Kapoor R, Muruganandham K, Gulia AK, Singla M, Agrawal S, Mandhani A, Ansari MS, et al. Predictive factors for mortality and need for nephrectomy in patients with emphysematous pyelonephritis. BJU Int. 2010;105(7):986-989. 